\title{
Risk management of biosimilars in oncology: each medicine is a work in progress
}

\author{
Arnold G. Vulto • Stacy A. Crow
}

Received: 5 July 2011 / Accepted: 1 August 2011 / Published online: 25 January 2012

(C) The Author(s) 2012. This article is published with open access at Springerlink.com

\begin{abstract}
Drug licensing and drug safety monitoring for standard chemical entities have been established and are routinely used. These have resulted in a solid foundation of knowledge from which confident therapeutic decisions can be made. For many chemical entities, this advanced level of experience is also present for the generic products. The expertise surrounding the development of biosimilar competitor versions is increasing and progress is encouraging. To address the reengineering and comparability complexities of biosimilars, the European Union imposed a requirement that risk management plans be included in the medications' marketing applications. This paper summarizes and discusses the circumstances complicating the public's view of drug safety, historical incidents during the transition from innovative to competitor products, as well as retrospective assessments of the development and postmarketing experiences thus far with two biosimilars. Through assessing the market entries and post-marketing experiences of biosimilars used in oncology, the healthcare field can better prepare for the next wave of comparator-products: biosimilar monoclonal antibodies.
\end{abstract}

Keywords Risk management · Drug licensing · Drug safety $\cdot$ Biosimilar $\cdot$ Monoclonal antibody $\cdot$ Oncology

\section{Introduction}

Discoveries surrounding disease states and their drug treatments are becoming increasingly complex. Conveying

This article is a revision of Vulto AG (2011) Risk management of biosimilars in oncology. Oncologie 13(5):196-200

A. G. Vulto $(\bowtie) \cdot$ S. A. Crow

Hospital Pharmacy, Erasmus University Medical Center,

P.O. Box 2040, 3000 CA, Rotterdam, The Netherlands

e-mail: a.vulto@erasmusmc.nl a risk-benefit ratio to patients was already a cumbersome task and as the medical field evolves, this task is becoming even more difficult. Many reasons lead to the discrepancy between the expectations of patients (a new, expensive drug will be better) and the harsh realities of physicians, other healthcare professionals and regulators. Many of these could be due to the public's lack of knowledge surrounding the complexity of pharmaceutical agents and the safeguarding of their use. Several of these discrepancies shall be discussed within this paper including: the process of drug licensing and why drug safety monitoring programs, like the creation of risk management plans, exist. Additionally, issues more closely related to the field of oncology itself, such as the development of biosimilars and use of monoclonal antibodies, are also covered.

\section{The safety myth of new drugs}

The development process for new medicines requires an extraordinary amount of time and work and all steps must be carefully planned. Looking past the complexity of pharmaceutical science itself, regulatory authorities that oversee the introduction of new drugs to the market require extensive documentation. The ability of innovators to foresee problems and work pro-actively to avoid these from happening is pivotal; avoiding risky situations when developing a newly innovative product will help simplified market-entry process.

Regulators require a minimum success standard prior to accepting claims that a drug is efficacious in a given population. Therefore, to circumvent compromising the new compound's reputation (and the profitability and shareholders' value), appropriate inclusion and exclusion criteria are determined to optimise the trial's patient population and design during the development phase. The 
main question that a new, innovative medication trial strives to answer could be summarized by some as: Will the drug do what it is designed to do in the smaller-sized test population of patients with a certain disease (with as few adverse side effects as possible)? By carefully selecting a patient population based on their personal health status (disease state status) and history, the trial is tailored to the target population. To some, this may seem like obfuscation of the true results; however, these conditions are necessary since the question-to-be-answered about the medication's efficacy is usually concerning treatments within certain patients. In many pre-licensing trials, many side effects may occur in a frequency not much different from that of placebos. It is an exciting time for innovators if their new medicinal agents overcome these obstacles and the medicines are granted market approval. They are then finally able to provide an answer to that previously contemplated central question by confidently stating: yes, this medication works and the potential side effects from it are worth the positive benefit seen from the desired mechanism in the trial population, but not necessarily in the real-life populations. This difference is often referred to in healthcare as a difference of efficacy (how well a medication works under ideal, controlled settings such as a trial) and effectiveness (how the medicine functions under real-world conditions).

As a new medication with an unscathed reputation enters the market, it brings with it the known knowledge gaps from the fine-tuned trials and these questions can only be answered through future use. However, once the drug is used in everyday conditions, outside the protective confines of the carefully selected study criteria, the safety in real life situations may vary when compared to the carefully selected trial population in terms of comorbidities, comedication, age, pregnancy etc. For some drugs, these new adverse effects are found quickly and tumultuous cases lead may prompt market removal of the medications $[1,2]$. For others, clarification of the risk-benefit ratio may take several years, as was the case in January 2010 with isotretinoine (Roaccutane ${ }^{\circledR}$ ). An uncommon, yet serious side effect of skin necrolysis associated with isotretinoine use was identified; the discovery of this possible side effect occurred 25 years after isotretinoine's initial introduction.

\section{A dilemma for regulators}

This uncertainty surrounding the actual, everyday risks of a medication's use within an all-encompassing, general population poses a dilemma for regulators. It is their job to safeguard the public against harmful side effects by thoroughly assessing the available data. Therefore, before a new drug enters the market, an evaluation procedure occurs to appraise safety data and effects, yet the tolerability of the drug by a more extensive populace has yet to be determined. Regulators prefer to not take risks, but at times, this is unfortunately unavoidable; conversely, hindering a medication's market entry could be interfering with a potentially life-saving treatment for some patients [3]. Every drug approval must go through this process of weighing the benefits and the risks, considering that, at times, those risks also include unknown outcomes that may surface later [4].

\section{Reactive reporting: pharmacovigilance}

To quickly and efficiently gain knowledge surrounding the lingering unknown safety data, post-marketing pharmacovigilance programs have been developed in all European Union (EU) countries. Healthcare professionals and, in some countries patients as well, are asked to report any unanticipated experience with a drug (including absence of an anticipated beneficial effect). The offices involved in this extended validation process confirm the unforeseen effects from an epidemiological perspective. From there, the results are forwarded to the pharmacovigilance database operate by the World Health Organization (WHO) Uppsala monitoring center. A better estimate of an actual risk of using the medication is then assessed, keeping in mind that these data reflect a more diversified patient population [5]. Based on the frequency of a side effect, how often it occurs (numerator), and the total known exposure, the number of patients known to have been treated in total (denominator), the results of the riskassessment is determined. This post-marketing process proves extremely useful in helping to detect less common side effects via mass collection. Although the pooling of new data helps to clarify the medication's risks when used at large, not knowing the exact number of patients exposed to the drug makes the "real" risk quantification yet another approximation; albeit a more accurate appraisal than was previously known [6].

\section{Pro-active reporting: risk management and risk minimisation}

One of the responsibilities of the drug designers is to consider side effects and risks that may occur after therapy with their innovative product. Since November 2005, the EMA (European Medicines Agency) has required a proposed management plan for almost all medicines they evaluate (exceptions are granted to some generic medications for which adequate safety data has already been demonstrated). After approval, this becomes part of the marketing license [7, 8]. 
The post-authorisation safety studies (PASS) or "phase IV studies" are intended to further detect side effects and quantify data with increasing meticulousness and fervour. They are completed by the marketing authorisation holder (MAH), who is responsible for reporting a predefined, large number of cases treated in everyday conditions. Phase IV studies provide the opportunity to further develop factors surrounding adverse effects: when, how and why they occur.

For drugs with intrinsic safety risks, defined in this paper as those with known adverse events associated with their class or the pharmacological action, further steps must be taken to minimise potential adverse effects in the patient population. For these medications, the EMA requires the applicant to submit another scheme in the form of a risk minimisation plan. Herein, the precautionary actions that have been taken to prevent patients from experiencing known adverse effects of the drug are explained. These adverse events can be either: a) those commonly seen in select groups of predisposed patients or b) those directly related to the pharmacological action of the drug in all patients, such as those designed to treat diseases of the blood and blood forming organs. The product erythropoietin is an example of a drug classified under the latter description. Simply stated, the goal of the risk management plan is to proactively establish a product's risk-benefit analysis, which enables a healthcare professional to make informed decisions.

Prescribing information is also dynamic and requires regular updating. As new information unfolds, the pharmaceutical company may be requested to update the known information for prescribers in the Summary of Product Characteristics (SPC). Those with prescriptive authority are encouraged to consult the latest version of the SPC available prior to making pharmaceutical treatment decisions. The most up-to-date reports about known side effects of a medicine should be considered before prescribing, thus making careful dating of the drug information essential for the protection of patients against known risks. From this perspective, each medicine has to be regarded as a work-in-progress.

\section{The results of these pharmacovigilance activities}

When such time, effort and money have been devoted to assessing the safety data of an innovative medication, the outcomes of these efforts are undoubtedly useful to inspect. Post-marketing studies on drug safety policies and risk management plans are scarce, but have begun to appear. The results of one such study regarding biopharmaceuticals indicated that the knowledge-base surrounding biologicals is comprised primarily from clinically relevant side effect data collected 7-8 years after market approval, not those noted in pre-approval trials [9]. In this study the medications used were approved in the European Union from 1995 to 2007 and "clinically relevant side effects" were considered to be those for which the prescribing information had to be altered. This could be done via a "dear doctor letter" or other direct communication with healthcare personnel.

Based on this study and others like it, the interpretation of the data could be as follows: when a drug has been marketed for 10 years or longer, the vast majority side effects have surfaced [8-11]. Conversely, there are most definitely cases where this proves not true [12]; however, in both cases, risk management strategies for the whole life cycle of a drug are of the utmost importance. Lastly, historical evidence shows that changes in manufacturing processes or source of raw materials at any stage of a medicine's development may cause numerous side effects to present suddenly; this was exemplified during the case with the adulterated heparin from China in 2009 [13]. These infrequent instances aside, it is encouraging to see that pharmacovigilance programs do, indeed, play a crucial role in disclosing the real-life safety profile of a drug throughout its lifecycle.

\section{Biosimilars}

It is valid to view the development and evolution of new pharmaceutical agents as an ongoing process. When a new drug is first developed, patent protection usually extends for approximately 20 years. On average, $8-10$ of these are used during the trials phases, leaving about 10-12 years of patent protection once the drug is marketed. When the patent has expired, competitors are allowed to produce medications with the same active ingredient-generic versions. If the copy-product meets quality criteria and pharmacokinetic bioequivalence, the competing drug is allowed to enter the market. This process has now occurred numerous times again for a vast variety of small-molecule medications.

Recombinant DNA technology drugs are, however, exceedingly more complex than small-molecule medicines. As patents for the recombinant medications began to expire, questions arose regarding the ability to produce duplications of these molecules exact enough to ensure patient safety. Innovators, wishing to protect their trade secrets, did not make available their production platforms (bacteria or cell cultures). Thus, these had to be generated anew by the competitor companies; a daunting task, indeed.

Moreover, the resulting product could not be precisely identical on account of the many steps involved in the downstream isolation, purification and subsequent formulation of the product, among other reasons. Small discrepancies would almost certainly occur and, even with 
achievement of the same pharmacological effect, the discrepancies could possibly lead to new adverse drug reactions (such as immunogenicity). Since these medicines were (and are) very similar, but may not be exact copies, the term "biosimilars" has been coined for these agents in Europe (in the United States "follow-on biologic medicines" is also used and in Canada "subsequent entry biologics").

\section{Eprex $^{\circledR}$ and the PRCA case}

The discussion surrounding the market entry of competitor versions of recombinant medications was intensified followed an unfortunate incident with Johnson \& Johnson/OrthoBiotech. In 1998, a reformulation of the innovative erythropoietin alpha product $\left(\right.$ Eprex $\left.^{\circledR}\right)$ was attempted. The original product was made with human serum albumin as a solubiliser and stabiliser. This ingredient was viewed by EU regulators as an undesirable excipient to which improvement could be made due to its potential to transmit bovine spongiform encephalopathy (BSE) made. Following the nearly worldwide introduction of the modified formulation, an increased rate of pure red cell aplasia was noted. The incidences of this immunological reaction against endogenous erythropoietin were less frequent with other erythropoietin formulations, including the non-modified Eprex ${ }^{\circledR}$ in the U.S. Correlations where then made between the increased occurrences of this once uncommon side effect and the new formulation of Eprex ${ }^{\circledR}$. Although the debate surrounding what specifically was responsible for the immunological reaction has yet to be solved, to many the lesson learned was undeniably transparent: manipulating the formulation may put patients at risk.

This case hastened the regulators to quickly address pleas from the innovative industry - biotech products are unique and require a different set of rules than that applicable to small molecule/simple-chemical generics. This was compatible with a process already addressed in 1996 and under development within Europe since 1998. Through a refined regulatory system, the evaluation and approval of competitive versions of recombinant medicines was started.

In terms of how this affected market-entry of these biosimilars, the unfortunate experiences with PRCA and erythropoietin were pivotal. It was determined that biosimilars also have the potential to cause to rare side effects and would consequently be required to prepare risk management guidelines prior to use [7]. This increase in safety regulations accommodated the European Commission's desire to safeguard patients against the unknown risks that new copies of recombinant molecules. While the new availability of highly specific medications at lower costs was also highly beneficial to improving patient care in terms of cost-savings.

\section{The risk management system devised by EMA for new medicines}

Currently, an EU Risk Management Plan must be submitted to the EMA as part of a marketing application for all new chemical entities and biosimilars. This process began in November 2005 and aimed to protect patients from harmful events. By determining that the benefits of a medicine exceed the risks by the greatest achievable margin, all patients are safer. A risk management plan consists of two parts:

In part I, the safety profile of the medication is described and pharmacovigilance activities proposed. Examples of these activities include collecting of spontaneously reported adverse events or post-authorisation safety studies. The goal being the continuation of studying the safety concerns of the medications following their usage in real-world situations [9]. Part II should evaluate the necessity for risk minimization activities and provide an action plan for each potential safety concern. The legal requirements for these plans are described in guidelines [7] and have recently been reviewed [14]. Prescribers are able to read these drug assessments created by the Committee for Medicinal Products for Human Use (CHMP) and proposed risk management plans. These documents are available in the European Public Assessment Report (EPAR), on the website of the EMA and via national drug licensing authorities.

Adding further to the necessary complexities surrounding risk management plans, a distinction is made within the plan itself between routine activities (i.e., being part of good medical practice) and additional measures. Supplementary measures can take many forms. Some examples are: providing specific details in the prescribing information (such as warnings), the production of educational leaflets, establishment of a specialized training program, the initiation of additional clinical studies, or planning longterm follow-up of treated patients. The results of all these extra activities must be included in confidential Periodic Safety Update Reports (PSURs) to the EMA.

\section{Lack of transparency}

Despite of the huge amount of work that must go into the preparation for and reporting of side effects, the process still operates as a black box to the outside world. Drug companies bear the formidable task of vigilantly reporting, yet healthcare providers, including prescribing physicians, do not have access to the actual risk management plans or the reports they contain. Detailed safety information should be a relevant factor when choosing to prescribe a drug. After being provided safety data from the market authorisation holders, the EMA internally analyzes and collects the safety 
reports in the PSURs; however, these are confidential documents, not accessible to outsiders. The French critical medicines review journal La Revue Prescrire filed a complaint on August 30, 2010 against the European Medicines Agency (EMA), demanding increased transparency to medicines' safety information and documentation. EMA is now considering providing wider access to this kind of documentation, beginning with specific requests for select situations $[15,16]$.

The SIGNAL-publications from the Uppsala Monitoring Centre of the WHO could provide another source of information. These reports which contain the results of post-marketing surveillance and other alerts are distributed to national pharmacovigilance authorities and the relevant pharmaceutical companies; yet they are, once again, not accessible to healthcare providers. Frau et al. [8] discuss similar concerns about the unavailability of the most significant risk management plan data. Per Frau et al., in minimum, the planned-pharmacovigilance-activities data should be made publically available, including information such as full-study protocols, clear timelines for their development and the information regarding doctor and patient programs [16].

\section{Experiences with biosimilars in oncology}

\section{Historically}

Two types of biosimilars of particular interest to the field of oncology are filgrastim $\left(\mathrm{G}-\mathrm{CSF}^{\circledR}\right)$ and erythropoietin. Filgrastim is a relatively small protein drug (175 amino acids, 19.6 kDalton) and has a strong safety record. It is a drug considered to be relatively safe in terms of immunogenicity [17], but it has commonly been used in immunocompromised patients and, therefore, less immunogenic responses are to be expected. When used outside this populace, neutralising antibodies can occur. When these antibodies are produced, the effectiveness of the drug is compromised. This immunologic response is not always easily detectible and makes the "normal" effects of G$\mathrm{CSF}^{\circledR}$ variable and a diverse range of outcomes possible. As a result, the identification of clinically relevant differences between an unpredictable innovator product and the biosimilar form of filgrastim is highly complex and the lack of efficacy is not always obvious. Precautionary measures have, thus, been taken within the licensing package for filgrastim and other biosimilars. The responsibility to monitor the occurrence of neutralising antibodies is listed within the package and to do so is the job of the prescriber. Currently available biosimilar filgrastim molecules have been licensed by the EMA after comparison with Neupogen ${ }^{\circledR}$ and are marketed by several companies under a plethora of names. In order of EMA licensing, these are: Filgrastim $^{\circledR}$, Ratiopharm ${ }^{\circledR}$, Biograstim ${ }^{\circledR}$, Ratiograstim ${ }^{\circledR}$, Tevagrastim $^{\circledR}$, Hexal $^{\circledR}$, Zarzio $^{\circledR}$ and Nivestim ${ }^{\circledR}$. Thus far, these biosimilar agents have been used with predominantly great success.

Erythropoietin is a larger and more complicated molecule (165 amino acids with heavy glycosylation, resulting in a molecular weight of $34 \mathrm{kDalton}$ ) and is used more frequently than filgrastim in patients with active immune systems. Resultantly, erythropoietin has the propensity to cause immunologic reactions. As such, PCRA and other immunerelated adverse events are key issues to be included in the required risk management plan. Immunogenicity, however, is not a solitary concern with erythropoietin; additional side effects relevant to this class of medications as a whole must also be addressed for both innovator and competitive products alike. Such class-common effects include consequences such as hypertension and thromboembolic events, the latter of which received particular attention in the field of oncology.

Oncologists struggled to help patients cope with the anaemia and fatigue that often accompany cancer and chemotherapy. They hoped that by occasionally administering additional injections of erythropoietin, erythropoiesis would be stimulated and blood transfusions could be avoided. Unfortunately, undesirable sequalae were also possible with two potential adverse outcomes of utmost concern. First, the effect of erythropoietin had other growth-stimulating properties, such as tumour growth. Second, the incidence of venous thromboembolism and a small unexplainable increase in mortality when anaemic cancer patients were treated with epoetins [18].

The increased incidence of tumour-growth among erythropoietin users was hardly discernable and the studies attempting to prove this effect contained sources of bias. Addressing the second outcome of possible thromboembolism, the increased incidence and mortality appeared to be dose-dependent and would found to be rarely problematic if the hemoglobin concentrations were not driven higher than $12 \mathrm{~g} / \mathrm{dl}$ [19]. Nevertheless, these side effects have been the subject of several studies and debates, both in Europe and the US [20]. In an attempt to better protect patients via openly displaying known safety data, the product information of all epoetins - innovative and competitor alike - were updated. The molecules currently approved by the EMA as biosimilar forms of erythropoietin are: epoetin alfa (marketed as Epoetin Alfa Hexal ${ }^{\circledR}$, Abseamed ${ }^{\circledR}$ and Binocrit $\left.{ }^{\circledR}\right)$; epoetin theta (marketed as Eporatio ${ }^{\circledR}$ and Biopoin ${ }^{\circledR}$ ) and epoetin zeta (marketed as Silapo ${ }^{\circledR}$ ).

\section{Outcomes}

The European Society of Medical Oncology (ESMO) has created an evidence-based practice guideline [21, 22] from 
the currently available risks-and-benefits data on the use of erythropoietin in cancer. Many of the negative outcomes following the use of erythropoietin in cancer patients were caused by the use of erythropoietin outside the approved indications and/or recommended dosage [10, 23]. When considering the use of erythropoietin in a patient with cancer, it should be noted that erythropoietin does not improve the outcome of the underlying disease; it is considered to be supportive and is aimed at improving only the quality of life, not mortality.

\section{Outlook}

The current experiences with the risk management of biosimilars in oncology have not led to unsurpassable safety concerns. These historical successes together with continuous surveillance, provide a safe foundation for confident prescribing of these medicines. It can also be said that the EMA regulators have put forth a solid effort to assure comparability between innovator products and biosimilars.

Building from these historical events and experiences with small molecules such as filgrastim and erythropoietin, regulators face the next challenge ahead: biosimilar monoclonal antibodies [24]. Monoclonal antibodies are larger structures with between 1,000 and 1,500 amino acids creating a mass of about 150 kDalton and comparability exercises for biosimilar monoclonal antibodies will be challenging [25]. In preparation, the EMA's Similar Biological Medicinal Products Working Party has drafted two guidelines to inform the pharmaceutical industry how the EMA plans to handle this new generation of biosimilars $[26,27]$. In the coming years, some patents of widely prescribed monoclonal antibodies used in oncology will begin to expire and guidelines such as these are paving the way for biosimilar versions of these medicines.

\section{Conclusion}

With the population increasing, costs of medications rising and treatment complexities intensifying, cost-escalation of drug treatments is becoming unavoidable. With conventional medicines, the use of competitive formulations is an effective way to reduce costs while maintaining effectiveness and access to drugs. For the more expensive protein drugs, the European Union has designed a biosimilar pathway to allow market access for competing versions of these medications. Due to concerns about the comparability, similarity and safety of these medicines, all biosimilars shall follow a post-authorization risk management system. This will help to inform regulatory authorities about the effects of biosimilars under practice conditions in a large- scale, diversified population. While transparency of the information reported could be improved, until now, no new or shocking findings have surfaced. Based on these experiences, the currently licensed biosimilars can be seen as equally efficacious and safe as innovator products when making prescribing decisions. With patents for monoclonal antibodies are expiring, we can look confidently ahead to the approval and introduction of a new generation of biosimilars.

Acknowledgement We wish to thank Hans Ebbers, M.Sc., Utrecht University, Department of Pharmacoepidemiology, for his contributions and assistance with the preparation of this article.

Conflict of interest statement The authors do not have any conflicts of interest to declare.

Open Access This article is distributed under the terms of the Creative Commons Attribution Noncommercial License which permits any noncommercial use, distribution, and reproduction in any medium, provided the original author(s) and source are credited.

\section{References}

1. Jüni P, Nartey L, Reichenbach S, Sterchi R, Dieppe PA, Egger M (2004) Risk of cardiovascular events and rofecoxib: cumulative meta-analysis. Lancet 364:2021-2029

2. Sayburn A (2010) Withdrawal of sibutramine leaves European doctors with just one obesity drug. BMJ 340:c477

3. Carpenter D, Ting MM (2005) Essay: the political logic of regulatory error. Nat Rev Drug Discov 4(10):819-823

4. Miller HI, Henderson DR (2007) Governmental influences on drug development: striking a better balance. Nat Rev Drug Discov 6(7):532-539

5. Goldman SA (1998) Limitations and strengths of spontaneous reports data. Clin Ther 20(Suppl C):C40-4

6. Vandenbroucke JP (2008) Observational research, randomised trials, and two views of medical science. PLoS Med 5(3):e67

7. CHMP (Committee for Medicinal Products for Human Use) (2005) Guideline on risk management systems for medicinal products for human use. European Medicines Agency document EMEA/CHMP/96268/2005 (accessible via www.ema.europa.eu)

8. Frau S, Font Pous M, Luppino MR et al (2010) Risk management plans: are they a tool for improving drug safety? Wur J Clin Pharmacol 66:785-790

9. Giezen TJ, Mantel-Teeuwisse AK, Straus SM et al (2009) Evaluation of post-authorization safety studies in the first cohort of EU risk management plans at time of regulatory approval. Drug Saf 32:1175-1187

10. Gascón P (2008) Safety update on erythropoiesis-stimulating agents: trials within and outside the accepted indications. Oncologist 13(Suppl 3):4-10, Review

11. Giezen TJ, Mantel-Teeuwisse AK, Straus SM et al (2008) Safety related regulatory actions for biologicals approved in the United States and the European Union. J Am Med Assoc 330:1887-1896

12. Mol PG, Straus SM, Piening S, de Vries JT, de Graeff PA, Haaijer-Ruskamp FM (2010) A decade of safety-related regulatory action in the Netherlands: a retrospective analysis of direct healthcare professional communications from 1999 to 2009. Drug Saf 33(6):463-474. doi:10.2165/11532840-000000000-00000 
13. Kishimoto TK, Viswanathan K, Ganguly T, Elankumaran S, Smith S, Pelzer K et al (2010) Contaminated heparin associated with adverse clinical events and activation of the contact system. N Engl J Med 362:1056

14. Zuniga L, Calve B (2010) Biosimilars: pharmacovigilance and risk management. Pharmacoepidemiol Drug Saf 19:661-669

15. Barbui C, Baschirotto C, Cipriani A (2011) EMA must improve the quality of its clinical trial reports. BMJ 25:342

16. Gøtzsche PC, Jørgensen AW (2011) Opening up data at the European Medicines Agency. BMJ 342:d2686

17. Thorpe RT, Waxhaw M (2006) Protein therapeutics and their immunogenicity. Eur J Hosp Pharmacy Practice 12(5):17-18

18. Bennet CL, Silver SM, Djulbegovic B et al (2008) Venous thromboembolism and mortality associated with recombinant erythropoietin and darbepoetin administration for the treatment of cancer associated anemia. J A Med Assoc 299:914-924

19. Dicato M (2008) Venous thromboembolic events and erythropoiesis-stimulating agents: an update. Oncologist 13(Suppl 3):11-15

20. Dicato M, Lawny L (2009) Concerns about erythropoiesis stimulating agents in oncology: an update. Eur J Hosp Pharm Pract 15(1):25-27
21. Schrijvers D, Roila F (2009) Erythropoiesis stimulating agents in cancer patients: ESMO recommendations for use. Ann Onc 20 (Suppl 4):iv159-iv61

22. Stark CG (2009) Risk management of ESAs in cancer patients: a role for the hospital pharmacist. Eur J Hosp Pharm Pract 15 (4):54-58

23. Glaspy JA (2009) Erythropoietin in cancer patients. Annu Rev Med 60:181-192. doi:10.1136/bmj.d2686

24. Schneider C (2008) Monoclonal antibodies-Regulatory challenges. Cur Pharm Biotechnol 9:431-438

25. Schneider C, Kalinke (2008) Toward biosimilar monoclonal antibodies. Nat Biotechnol 26:985-990

26. CHMP (Committee for Medicinal Products for Human Use) (2010a) Draft guideline on immunogenicity assessment of monoclonal antibodies intended for in vivo clinical use. European Medicines Agency document EMEA/CHMP/BMWP/403543/2010 (version 18 November 2010, accessible via www.ema.europa.eu)

27. CHMP (Committee for Medicinal Products for Human Use) (2010b) Draft guideline on similar biological medicinal products containing monoclonal antibodies. European Medicines Agency document EMEA/CHMP/BMWP/86289/2010 (version 18 November 2010, accessible via www.ema.europa.eu) 